\title{
Evaluation of halitosis and sialometry in patients submitted to head and neck radiotherapy
}

\author{
Danielle-Frota Albuquerque ${ }^{1}$, Elen-de Souza Tolentino ${ }^{2}$, Flávio-Monteiro Amado ${ }^{3}$, Cazuo Arakawa ${ }^{4}$, Luiz- \\ Eduardo-Montenegro Chinellato ${ }^{5}$
}

\footnotetext{
${ }^{1}$ MSc in Stomatology at Bauru Dental School, University of São Paulo, Bauru-Brazil

${ }^{2}$ Graduate student (PhD degree) at Bauru Dental School, University of São Paulo, Bauru-Brazil

${ }^{3}$ Phd in Stomatology at Bauru Dental School, University of São Paulo, Bauru-Brazil

${ }^{4}$ Radiotherapeutic doctor from Manoel de Abreu Hospital , Bauru -São Paulo -Brazil

${ }^{5} \mathrm{PhD}$ Professor at Bauru Dental School, University of São Paulo, Bauru-Brazil
}

Correspondence:

Faculdade de Odontologia de Bauru-USP

Departamento de Estomatologia

Alameda Dr. Octávio Pinheiro Brisola, 9-75

Vila Universitária - CEP 17012-901 Bauru - SP - Brazil

elen_tolentino@hotmail.com

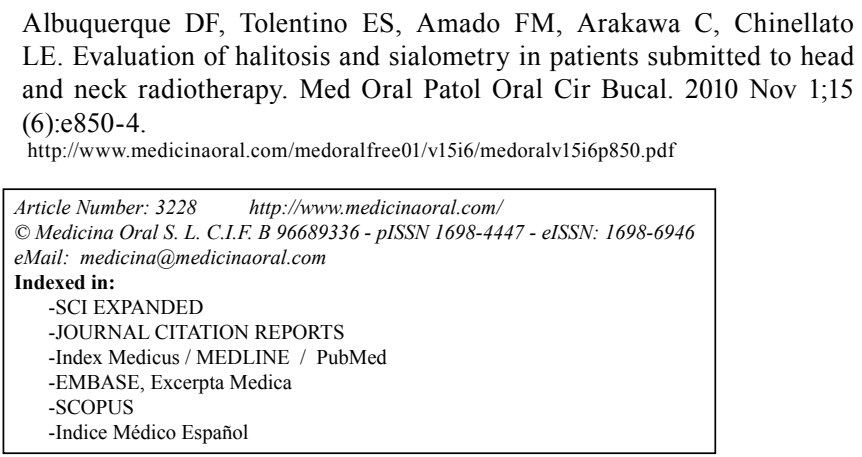

\begin{abstract}
Objectives: The aim of this study was to investigate the parameters of halitosis and sialometry in patients who had undergone head and neck radiotherapy, correlating oral concentration of volatile sulfur compounds (VSC) with the presence of tongue coating, salivary flow rate and BANA test.

Material and Methods: 35 patients allocated in two groups were examined: group I (control) - patients with systemic and oral health; group II - patients submitted to head and neck radiotherapy. All volunteers were submitted to halitosis measurements through a sulphide monitor, evaluation of tongue coating weight, non-stimulated sialometry and BANA test.

Results: The results were analyzed through analysis of variance, Pearson's correlation and Student's t-test, showing that there was statistically significant difference in halimetry between the groups, where the irradiated patients showed halitosis. There was a relation between the presence of tongue coating and the levels of VSCs in both groups and it was also noted that the irradiated patients showed a decreased salivary flow rate compared to healthy patients.

Conclusion: On the conditions of the present research, it was concluded that halitosis can be considered an adverse effect of radiotherapy, connected to hiposalivation and poor oral health.
\end{abstract}

Key words: Halitosis, head and neck cancer, radiotherapy, saliva, tongue. 


\section{Introduction}

Surgical therapy was long the primary treatment modality for patients with head and neck cancer. Postoperative radiotherapy has been considered the standard of care for a subset of patients for many decades (1). The adverse effects of head and neck radiotherapy, acute or chronic, have a determinant impact on the quality of the patient's life (2). These effects are very important for the dental surgeon, who has a fundamental role in preventing and reducing them. Changes resulting from irradiation occur mainly in mucosa, jaw bones, salivary glands and teeth (3). Because of the salivary glands are radiosensitive, the atrophic and acinar degeneration caused by radiotherapy commonly result in the decrease of saliva production $(4,5)$.

Halitosis is highly connected to the amount of saliva (6), which can be another adverse effect that interferes in the quality of life and socialization of the irradiated patients $(7,8)$. Literature shows an association of hiposalivation with the adverse effects from radiotherapy $(9,10)$. However, the association between halitosis and radiotherapy is less known.

The aim of this study was to establish a correlation between halitosis and head and neck radiotherapy, through evaluation of halitosis and sialometry in patients who had undergone radiotherapy when compared to healthy and non-irradiated individuals.

\section{Material and Methods}

This study was approved by the Human Research Ethics Committee of Bauru Dental School, University of São Paulo - Brazil (process no 104/2005) and is in accordance with the Helsinki Declaration of 1975, as revised in 1983. All volunteers signed an informed consent document. The study was conducted over one month period, with one or two attendances per day. The sample consisted in 35 patients, divided into two groups: Group I (control): 10 patients who have recently concluded dental treatment in Bauru Dental School, with entire oral health; Group II: 25 volunteers selected among patients from Manoel de Abreu Hospital, referenced for cancer treatment in the city of Bauru - Brazil. The sample size was justified by the fact of many patients have not finished periodontal and/or radiotherapic treatment at the moment of the consultations. Anyway, the sample was appropriated to the utilized statistical tests.

Patients from group II were submitted to radiotherapy for treatment or complementary treatment for head and neck tumors. The radiation area covered at least one of the major biggest salivary glands or part of them. The radiotherapy treatment of all the patients had already been concluded and they were being monitored from 1 to 6 months after the last session of radiotherapy.

All patients underwent two appointments, which were conducted by the same examiner. In the first one, pri- or to the clinical examination, all subjects were asked to fill out a questionnaire about personal information, general and oral health. In this consultation the patients received the following guidelines, for the procedures to be carried out in a further moment, such as: 24 hours earlier, avoid spicy and/ or flavored food; 3 hours earlier, avoid brushing the teeth, using dental floss, chewing gum, drinking alcoholic drinks, smoking and, at the moment of the appointment, not being using perfume. The following stages were performed in a second appointment.

\section{Halimetry}

The halimetry values in all individuals were obtained through a sulphide monitor (Halimeter - Interscan Corporation, Chatsworth, USA). Before measurements, patients were kept seated, relaxed and without talking for 1 minute, with their mouth shut. A disposable plastic tube connected to the monitor was introduced $4 \mathrm{~cm}$ inside the patient's mouth. The patients were instructed to bring their mouth slightly opened on the tube without inhaling or exhaling during the analysis. Those measures were taken three times, which resulted in a final average.

2. Evaluation of tongue coating and new measures of halimetry

After removing the excess of humidity of the tongue with gauze, the tongue coating was removed with a tongue cleaner and was placed in a filter paper previously weighted. The material was stored for 24 hours for drying and weighing. The tongue coating weight was obtained through a balance of accuracy (A \& D Company Limited, Tokyo). After removing the tongue coating, halimetry was performed again, in order to identify possible variations in the VSCs concentrations.

3. BANATM Test (benzoyl-DL-arginine-napthylamide) The test was performed to identify a possible relation between the presence of microorganisms BANA positive and the values of halimetry. A small amount of tongue coating was collected and transferred to the BANA test strip (BANAMet LLC - Ann Arbor, MI, USA). A drop of distilled water was poured on the reagent strip, which was folded and kept this way with a paperclip, so that the reagent would be in contact with the organic material, remaining 24 hours in that situation, as indicated by the manufacturer. After that period the reaction was noted and the result obtained, classified as negative and positive, according to the obtained color.

\section{Sialometry}

After remaining 5 minutes at rest, with the eyes opened, without stimulating salivation, each patient spit out all saliva obtained in that period in a recipient graded in millimeters (ml) - non-stimulated salivary flow. After this procedure, each patient was given a piece of sterile hyperboloid which was used to stimulate salivation through mastication for 5 minutes, and the saliva obtained was also placed in a recipient graded in $\mathrm{mL}-$ 
stimulated salivary flow.

\section{Protocol of radiotherapy treatment}

The radiotherapy treatment protocol was the same for all patients from group II. The patient wore a facial mask for beam radiation collimation, molded individually. The device Theratron 80 (Atomic Energy of Canada Limited) was used for the treatment. The total dosage of radiation varied from $5040 \mathrm{cGy}$ to $6480 \mathrm{cGy}$, with an average of $5463.2 \mathrm{cGy}$, fractioned in a daily dosage of $180 \mathrm{cGy}$, and the days alternating for each patient. All the patients received radiation in two areas, supraclavicular fossa and opposite parallel. Size and depth of the area of incidence of beam radiation had a slight variation for each case, depending of the features of the tumor. The dosage and fractionation of the treatment were individual for each case and decided by the medical team. All selected patients have had at least one of the major salivary glands involved in the area of radiation.

\section{Statistical analysis}

The results were analyzed by using ANOVA test, in the variables which quantified halitosis before and after removing tongue coating. When there was significant difference, we performed Tukey test. The relation among oral halimetry, dry weight of tongue coating and salivary flow with or without stimulation were analyzed through Pearson's correlation test. In order to verify the correlation between halimetry and BANA test, Student's " $t$ " test was used. For all the analyses, a 5\% significance level was undertaken $(p<0.05)$. The computer software used was Microsoft Office Excel 2007.

\section{Results}

Tumors that affected group II were squamous cell carcinoma $(\mathrm{n}=22)$ or adenocarcinoma $(\mathrm{n}=3)$ in different areas: larynx $(n=6)$, pharynx $(n=4)$, parotid $(n=4)$, tonsillar fossa $(n=3)$, tongue $(n=3)$, jugal mucosa $(n=2)$, floor of the mouth $(\mathrm{n}=3)$.

Group I presented lower initial halimetry average (65.133 ppb) when compared to group II (143.747 ppb). The values of initial halimetry were statistically greater than the ones of final halimetry in both groups $(p<0.001)$ (Table 1). The dry weight of tongue coating in group II $(0.016 \mathrm{mg})$ was smaller than in group I $(0.0179 \mathrm{mg})$, however that difference was not significant (Table 1). Group II showed a decrease in salivary flow, in stimulated and non-stimulated sialometry, with a statistically significant difference $(p<0.005)$ (Table 1).

In the evaluation of the correlation between the studied variables and halitosis, in group I this correlation was noted between initial halimetry and presence of tongue coating and between stimulated and non-stimulated salivary flow $(\mathrm{p}<0.005)$. Group II, showed a significant correlation between initial and final halimetry, between initial halimetry and presence of tongue coating, and between stimulated and non-stimulated salivary flow $(\mathrm{p}<0.05)$.

In both groups the number of negative BANATM tests $(n=25)$ was greater than positive results $(n=23)$, with a statistically significant difference among the negative and positive results and halimetry in group I and when the entire sample was together $(\mathrm{p}<0.005)$ (Table 2).

Table 1. Values of mean (SD) of groups I and II regarding the variables IH, FH, TC, NSSF and SSF.

\begin{tabular}{|cccc|}
\hline Variables & Group I & Group II & p value \\
\hline IH & $65.133 \pm 28.03$ & $143.747 \pm 62.092$ & $0.000^{*}$ \\
\hline FH & $50.4 \pm 24.95$ & $123.453 \pm 49.670$ & $0.000^{*}$ \\
\hline TC & $0.0179 \pm 0.007$ & $0.016 \pm 0.017$ & $\mathrm{~ns}$ \\
\hline NSSF & $3.33 \pm 1.365$ & $1.448 \pm 1.015$ & $0.002^{*}$ \\
\hline SSF & $8.46 \pm 2.307$ & $2.548 \pm 1.660$ & $0.000^{*}$ \\
\hline
\end{tabular}

*- statistically significant difference $(\mathrm{p}<0.05)$; ns - non statistically significant difference IH initial halimetry; FH- final halimetry - after removing the tongue coating (ppb); TC - dry weight of tongue coating $(\mathrm{mg})$; NSSF - non-stimulated salivary flow $(\mathrm{mL})$; SSF- stimulated salivary flow $(\mathrm{mL})$

Table 2. Student's " $\mathrm{t}$ " test for comparison between halimetry and positive and negative BANATM test groups.

\begin{tabular}{|lcccc|}
\hline & Negative & Positive & & \\
\hline & mean \pm sd & mean \pm sd & T & p \\
\hline All & $104.1 \pm 51.2$ & $157.3 \pm 67.1$ & -3.10 & $0.003^{*}$ \\
\hline Group I & $47.0 \pm 9.4$ & $92.2 \pm 23.9$ & -4.25 & $0.002^{*}$ \\
\hline Group II & $128.1 \pm 45.6$ & $167.1 \pm 77.6$ & -1.58 & 0.126 \\
\hline
\end{tabular}

*- statistically significant difference $(\mathrm{p}<0.05)$ 


\section{Discussion}

In the present study, the use of the sulphide monitor enabled the evaluation only of the relation between VSCs and halitosis. That represents a limitation of methodology since bad breath consists of other volatile organic compounds and other gases (11).

Regarding oral halimetry, group I presented lower initial average when compared to group II, as demonstrated in table 1 . This can be justified because group I was free of oral problems, since the measures of halimetry were performed immediately after the end of multidisciplinary dental treatment. Those data confirmed the ones from Scully et al. (12), which affirm strict relations between halitosis and oral health.

As shown in table 1 , the values of initial halimetry were statistically greater than the ones of final halimetry in both groups. Final halimetry was performed after tongue cleaning. This result confirms the hypothesis that tongue coating is an important cause of bad breath and also that cleaning the tongue properly must be part of daily oral hygiene (13). Waler (14) showed that the largest production of VSCs is in the tongue dorsum. Yaegaki and Sanada (15) noticed that removing tongue coating reduced in $50 \%$ the production of VSCs. De Boever and Loesche (16) evaluated the amount of tongue coating visually and noticed that the score of oral breath was highly connected to the odor of the tongue, presence and extension of coating.

Seemann at al. (17) noted that the tongue cleaner showed a higher percentage of reduction of VSCs than the tooth brush. That fact justifies the use of tongue cleaner in the methodology of this study, besides the tongue cleaner is easy to use and causes fewer stimuli to nausea reflex. In our study, the amount of tongue coating was evaluated quantitatively after drying, confirming methodologies used by other authors $(15,18)$. The dry weight of tongue coating in the irradiated patients was smaller than in the healthy patients, however that difference was not significant.

Regarding to salivary flow, all patients in group II showed a decrease in the amount of saliva, in stimulated or non-stimulated sialometry, with a statistically significant difference (Table 1). The value of $0.1 \mathrm{~mL} /$ min for salivary flow without stimulus was considered severe glandular hypofunction. There was a decrease in sialometry without stimulus from group II of $56.51 \%$ compared to group I. The BANATM test - an enzymatic method used as an indicator of the presence of microorganisms responsible for periodontal diseases - was performed in groups I and II. In both groups the number of negative tests $(\mathrm{n}=25)$ was greater than positive results $(\mathrm{n}=23)$. In table 2 , it is noted that there was a statistically significant difference among the negative and positive results and halimetry in group I and when the entire sample was together. Some authors have not noted rela- tion between the levels of VSCs and the results of lingual BANATM test. In the study of Monteiro-Amado et al. (18), there was not a relation between the value of BANATM test and the values of halimetry.

The irradiated patients showed halitosis when submitted to halimetry. According to Conceição et al. (19), halimetry above $100 \mathrm{ppb}$ can be considered as halitosis. Therefore, in the conditions of the present study, it is possible to conclude that halitosis can be considered an adverse effect from radiotherapy, connected with hiposalivation and poor oral health. For that reason, patients who had undergone or will be submitted to head and neck radiotherapy must always be checked by the dental surgeon, who has an important role in preventing and treating adverse effects caused by that procedure, and also trying to provide oral care to improve the quality of life of the irradiated patients.

According to the results obtained within the applied methodology, it is possible to conclude that halitosis was detected in irradiated patients and non-stimulated salivary flow was extremely reduced in these patients. Moreover, there was relation between presence of tongue coating and VSCs levels, with a decrease in the values of halimetry after removing tongue coating in both groups. We concluded that halitosis is an adverse effect of head and neck radiotherapy.

\section{References}

1. Ko C, Citrin D. Radiotherapy for the management of locally advanced squamous cell carcinoma of the head and neck. Oral Dis. 2009;15:121-32.

2. Vissink A, Jansma J, Spijkervet FK, Burlage FR, Coppes RP. Oral sequelae of head and neck radiotherapy. Crit Rev Oral Biol Med. 2003;14:199-212.

3. Sciubba JJ, Goldenberg D. Oral complications of radiotherapy. Lancet Oncol. 2006;7:175-83.

4. Bomeli SR, Desai SC, Johnson JT, Walvekar RR. Management of salivary flow in head and neck cancer patients--a systematic review. Oral Oncol. 2008;44:1000-8.

5. Pathak KA, Bhalavat RL, Mistry RC, Deshpande MS, Bhalla V, Desai SB, et al. Upfront submandibular salivary gland transfer in pharyngeal cancers. Oral Oncol. 2004;40:960-3.

6. Möller P, Perrier M, Ozsahin M, Monnier P. A prospective study of salivary gland function in patients undergoing radiotherapy for squamous cell carcinoma of the oropharynx. Oral Surg Oral Med Oral Pathol Oral Radiol Endod. 2004;97:173-89.

7. Van den Broek AM, Feenstra L, de Baat C. A review of the current literature on aetiology and measurement methods of halitosis. $\mathrm{J}$ Dent. 2007;35:627-35.

8. Tonzetich J. Production and origin of oral malodor: a review of mechanisms and methods of analysis. J Periodontol. 1977;48:13-20.

9. Llena-Puy $\mathrm{C}$. The rôle of saliva in maintaining oral health and as an aid to diagnosis. Med Oral Patol Oral Cir Bucal. 2006;1:E449-55. 10. Torres-Carranza E, Infante-Cossío P, Hernández-Guisado JM, Hens-Aumente E, Gutierrez-Pérez JL. Assessment of quality of life in oral cancer. Med Oral Patol Oral Cir Bucal. 2008;13:E735-41.

11. Van den Velde S, Quirynen M, Van Hee P, Van Steenberghe D. Halitosisassociated volatiles in breath of healthy subjects. J Chromatogr B Analyt Technol Biomed Life Sci. 2007;853:54-61.

12. Scully C, el-Maaytah M, Porter SR, Greenman J. Breath odor: etiopathogenesis, assessment and management. Eur J Oral Sci. 1997;105:287-93. 
13. Rosenberg M. Clinical assessment of bad breath: current concepts. J Am Dent Assoc. 1996;127:475-82.

14. Wåler SM. On the transformation of sulfur-containing amino acids and peptides to volatile sulfur compounds (VSC) in the human mouth. Eur J Oral Sci. 1997;105:534-7.

15. Yaegaki K, Sanada K. Volatile sulfur compounds in mouth air from clinically healthy subjects and patients with periodontal disease. J Periodontal Res. 1992;27:233-8.

16. De Boever EH, Loesche WJ. Assessing the contribution of anaerobic microflora of the tongue to oral malodor. J Am Dent Assoc. 1995;126:1384-93.

17. Seemann R, Kison A, Bizhang M, Zimmer S. Effectiveness of mechanical tongue cleaning on oral levels of volatile sulfur compounds. J Am Dent Assoc. 2001;132:1263-7.

18. Monteiro-Amado F, Chinellato LE, de Rezende ML. Evaluation of oral and nasal halitosis parameters in patients with repaired cleft lip and/or palate. Oral Surg Oral Med Oral Pathol Oral Radiol Endod. 2005;100:682-7.

19. Conceição MD, Marocchio LS, Tárzia O. Evaluation of a new mouthwash on caseous formation. Braz J Otorhinolaryngol. 2008;74:61-7.

\section{Acknowledgements}

This investigation was supported by CAPES. 